\title{
Supporting Contexts for Indoor Navigation using a Multilayered Space Model
}

\author{
Becker, T., Nagel, C., Kolbe, T. H. \\ Technische Universität Berlin, Strasse des 17. Juni 135, 10623 Berlin, Deutschland \\ \{becker;nagel; kolbe\}@igg.tu-berlin.de
}

\begin{abstract}
Indoor navigation highly depends on context and requires flexible data structures to support the many use cases and configurations. For example, an indoor navigation system must cope with different localisation techniques, infrastructures, and capabilities of mobile devices. Also physical constraints from the built-up environment, different modes of navigation (like walking, driving, or flying), and thematic restrictions like security zones have to be considered. In this paper we propose a novel modelling framework for indoor navigation which considers the aspects of route planning for different modes of navigation on the one hand and of various localisation techniques on the other hand. It is based on a structured and multilayered space model in which every type of physical or logical aspect is mapped within its own space layer. It is shown how layers can be combined according to concrete navigation contexts to build an npartite graph facilitating both route planning and localisation.
\end{abstract}

\section{Introduction}

Indoor navigation comprises route planning as well as localization and tracking of moving subjects or objects within interior built environments. Both aspects of indoor navigation depend to a great extent upon the context of navigation which is constituted by three main factors. A first factor is the mode of locomotion of the moving subject or object such as walking, driving a wheel chair or mobile robot, or even flying (e.g., quadrocopter). Each mode of locomotion requires a specific and separate partitioning of indoor space into navigable and non-navigable areas. For different modes of locomotion the resulting navigable areas may be disjoint, overlapping or equal. Second, the context of navigation is determined by the localization techniques and the localization infrastructure.
Amongst others, this comprises the methods used for localization and tracking, the positioning and ranges of sensors and transmitters, and the technical characteristics and capabilities of end-user devices. A third factor is logical contexts representing pre-knowledge or navigation constraints which result from specific application domains. For example, security zones or evacuation areas within buildings denote logical navigation contexts which influence the accessibility of navigable indoor spaces.

The context of navigation induces a configuration problem with a high degree of combinatorial complexity, because different localization techniques, modes of locomotion and logical zones can be combined arbitrarily. Current models for indoor navigation often reduce this complexity by tailoring the context of navigation to either one specific configuration or a limited subset. Accordingly, the interior built environment is mostly partitioned due to one mode of locomotion and corresponding route planning and addressing criteria on the one hand and a given localization technology and sensor characteristics on the other hand. Often a geometric route network for indoor navigation is proposed which maps the resulting subdivisions of indoor space to a graph structure representing topological connectivity. Further aspects of the fixed navigation configuration are introduced into this graph as a set of homogenous attributes for nodes and edges. While these approaches are well-suited for a single configuration they lack the flexibility to support multiple contexts such as additional modes of locomotion or different localization techniques.

In a recently published paper we have proposed a novel framework for route planning and localization/tracking within indoor navigation systems [1]. This framework allows for the integration of conceptually separated indoor space models within a multilayered representation. The layers are independent in such that they represent separate decompositions of indoor space according to different semantic criteria such as building topography or sensor characteristics. Additional layers may be added to denote the subdivision of indoor space 
according to different modes of locomotion or with respect to logical contexts. The different layers are then linked by joint-states which mutually constrain possible locations of moving subjects or objects in either space model.

In this paper we show how the multilayered space model (section 2 and 3) can be used to support multiple contexts (section 4) for indoor navigation and why subspacing the navigable space (sec. 4 and 5) is induced by the context or respectively by the mode of navigation. Finally, in section 6 we present the resulting data model and in section 7 we draw our conclusions and point to future work.

\section{The Multilayered Model}

While route planning requires models which reflect the internal structure of a building, localization techniques require complementary models reflecting the characteristics of sensors and transmitters. Since the partitioning of building space differs in both cases, a separation of different space models into a multilayered representation is proposed in Becker et al. [1]. The space which is represented by the model can be subdivided in two types of space: physical and logical. While physical space layers are qualified by physical conditions (built-up space, sensor coverage), the logical ones subdivide the space according to logical conditions (e.g. accessibility, security zones). Physical layers comprise, for example, the topographic space and its dependent subspace layers (e.g. mode of navigation) and sensor space. Sensor space is characterized by different localization techniques such as Wi-Fi or RFID which differ in signal propagation and signal coverage. For this reason, each of them is defined in its own layer. Examples for logical layers can be found in the field of security or emergency (e.g. zoning layers for security levels, danger zones, evacuation areas etc.).

Each layer within the multilayered model is systematically subdivided into primal and dual space on the one hand and in geometry and topology on the other hand. Lee $[2,3]$ and also Gold $[4,5]$ pointed out how indoor spaces can be modelled based on Poincaré duality [8]. In [1] it is shown how the approach of Lee [2,3] can be cast in a general framework which forms a starting point for the multilayered approach. A key aspect of the framework is the clear separation of different space models, i.e. topographic space and sensor spaces, as layers. Each layer is structured in a similar way according to the structured space model presented in [1].

Within the dual graph structure of one layer a node in dual space represents a space (e.g. a room within a building) in primal space and an edge in dual space represents the adjacencies or connections (e.g. doors or passages as intra-space connections). Fig. 1 shows an example of four rooms in topographic space and additionally two more space layers (one for Wi-Fi transmitters and the other one for RFID).

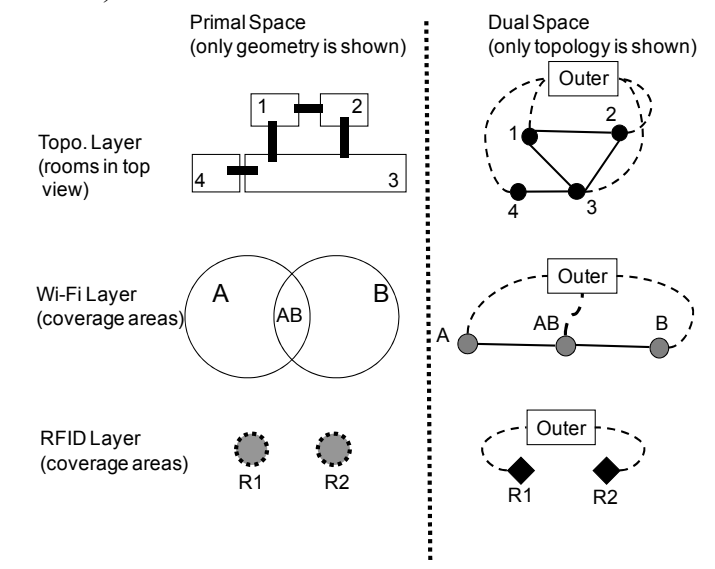

Figure 1. Example for topographic space and two different sensor spaces (Wi-Fi, RFID) in primal and dual space representation

All Object representations in primal space (left side of the figure) have their corresponding representations in dual space, shown on the right side of the figure. In the middle part of the figure $\mathrm{Wi}-\mathrm{Fi}$ transmitters and in the lower part of the figure RFID transmitter in primal and dual space are illustrated. Since the ranges of the Wi-Fi access points A and B overlap, the geometries representing the extent of signal coverage are partitioned accordingly into $\mathrm{A}, \mathrm{AB}$, and $\mathrm{B}$. This is required, because the range area $\mathrm{AB}$, for example, describes a different homogeneous area than $\mathrm{A}$ or $\mathrm{B}$. Within area $\mathrm{AB}$ a Wi-Fi device is able to receive the signal of two access points whereas in A or B only the signal of one access point can be received. To describe areas with no sensor coverage, an additional area called "outer" is defined for every layer. This area is needed when the navigating subject or object leaves e.g. the range of a sensor without other sensors around (see right side of fig. 1).

The context of navigation comprises physical, techni$\mathrm{cal}$, and logical aspects. Depending on the use case specific aspects have to be considered. In order to allow for a flexible combination, each aspect is modeled in a separate layer (see fig. 2). In contrast to existing models (e.g. see [7]) where space decompositions are influenced by other semantics, e.g., by the received signal strength of the transmitter or signal source (Wi-Fi fingerprinting), the multilayer approach facilitates individual modeling and changing of spaces without influencing other space representations.

\section{The Joint State}

The connectivity or adjacencies within each layer of the model constitute a graph structure in dual space (c.f. 
$[1,2,3,4,5])$. This dual graph represents cells (i.e., partitions of a space) as nodes and their (topological) adjacencies as edges. Furthermore, the nodes can be seen as possible states of a navigating subject or object while the edges denote state transitions, i.e. events caused by the movement of the subject or object according to the adjacency relations between the cells in primal space. Since a layer is based upon a disjoint partitioning of (Euclidean) space, a navigating subject or object can only be located in exactly one cell at a given point in time and thus only one state may be active. By this means, the dual graph of each layer also represents a state transition diagram.

Although each layer within the multilayered model describes a specific partitioning of space, the different space models cover the same real space. Therefore, a subject or object is at any given time exactly in one cell (or state) in each layer simultaneously. This overall state is thereby denoted by the active states of all space layers. However, only specific combinations of states from different layers are valid and can be active at the same time. The combinations are expressed by additional edges linking the nodes between different layers.

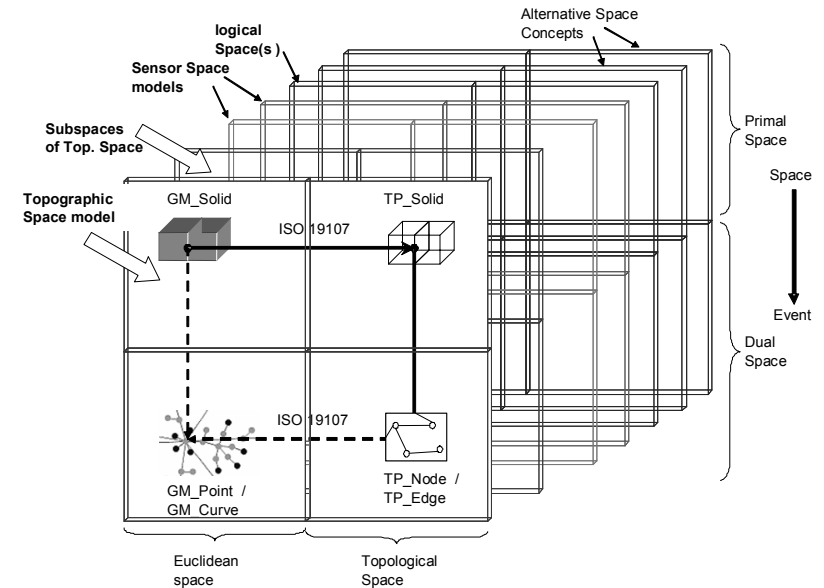

Figure 2. Multilayered combination of distinct space concepts

These so called joint edges are derived by pair wise intersecting the cell geometries from different layers. A joint edge between two such nodes is inserted if the intersection of the interior of the two corresponding cell geometries is non-empty. Therefore, the joint edges represent the Egenhofer relations "contains", "overlap" and "equals" [6] between two cells from different space layers and thus denote inter-space connections. Each tuple of nodes connected in pairs by joint edges is called a joint state. The following figure illustrates the dual graphs of three space layers together with their inter-space connections.

The joint state of navigation is exactly a clique of all active states of all space layers and is represented by a hyperedge within the graph structure. The hyperedge de- notes the n-ary relationship between all affected layers (see the bold grey line in fig. 3). In fig. 3, the cross in the upper part marks a possible position of a moving subject or object within a building. For this position, three interspace connections can be found which determine a ternary relationship or clique between the affected cells from the topographic space, the Wi-Fi sensor space, and the RFID sensor space. This hyperedge can be stored, for example, as a tuple in a table in an n-ary relation. For the sake of readability, the outer nodes are not included in fig. 3 , but nevertheless they also must be reflected.

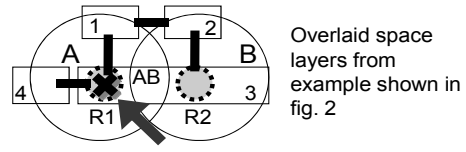

Location of a navigating subject in primal space is characterized by a hyperedge in the $n$-partite graph

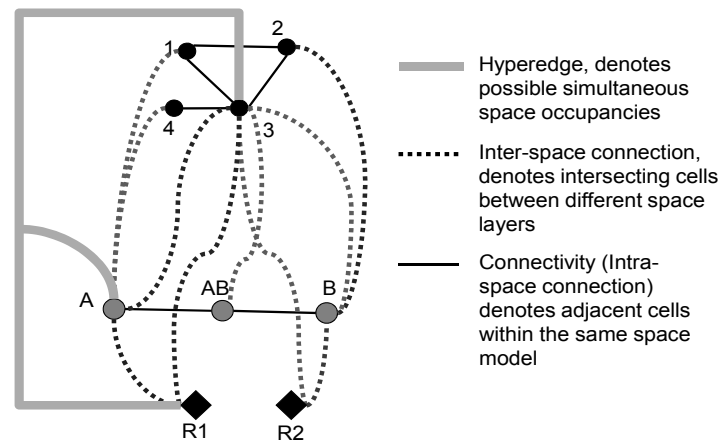

Figure 3. Resulting n-partite graph from example in fig. 2 with intra-space and inter-space connections. The hyperedge denotes a possible joint state. The incident nodes of a hyperedge build a clique of inter-space connections. Please note that the outer nodes have been omitted for the sake of readability.

The overall structure illustrated in this section constitutes an $\mathrm{N}$-partite graph, where all the nodes from all $\mathrm{N}$ layers are included but are separated into $\mathrm{N}$ partitions which are connected by the inter-space connections. Furthermore, the graph also contains the state transition edges (intra-space connections).

\section{Context as selection criterion}

Within the multilayered space model all contextual configurations can be represented as separate layers which are connected via the inter-space connection relation (see data model in section 5). The relevant data for indoor navigation or routing are selected and derived from the set of layers (n-partite graph, see fig. 4).

The selection is depending on logical or thematic considerations as well as on the existing localization device configurations. In fig. 4 an example for such a selection is shown. In the upper part the precomputed n-partite graph 
constituted by all different contexts is shown. In the lower part of the figure the user or application dependent selection of relevant space models from the set of layers (e.g. stored in a database) is shown. After the contextdependent selection the cliques are detected in order to identify all possible joint states (hyperedges). By intersecting all the geometries which are associated to the incident nodes (see section 3) of each hyperedge a 3D intersection volume results which constitutes the actual area in which the navigating subject or object must be in. The extent of this volume is a measure for the uncertainty of the absolute position of a navigating subject or object.

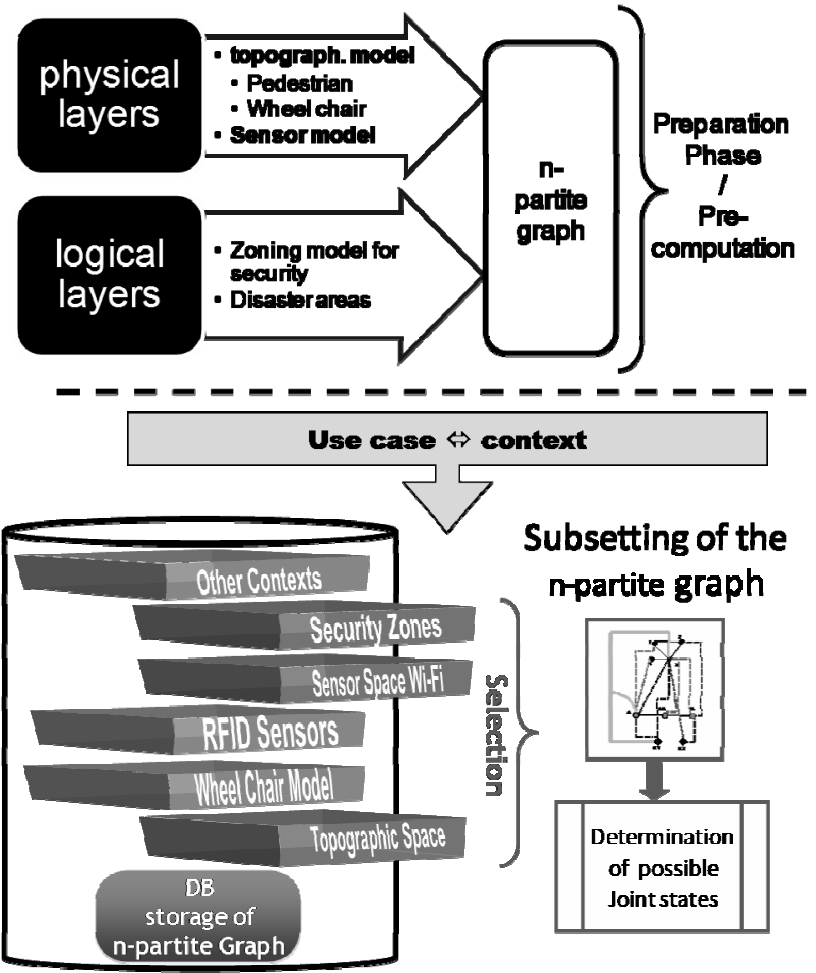

Figure 4. The actual use case defines the context. This is reflected by the selection of the according space models.

\section{Subspacing}

Cells within a space model (e.g. topographic space) may be subdivided due to specific considerations given by the mode of navigation. The topographic space is well defined and described by different existing standards like CityGML [11] or IFC [12] on a room (space) level, however, a decomposition by different contexts in differently smaller parts can occur. The crucial point is the decomposition may constitute a subset of the whole room and therefore a separation in one "Main space layer" and other additional context dependent layers is reasonable (see fig. 5). These decompositions of the topographic space are not independent of the higher level layer, because they reflect only a context specific partition of the higher level space. Therefore, the inter-space connection between a main layer and its subspacing layers is restricted to the topological relations qualified as "contains" / "inside" and equal.

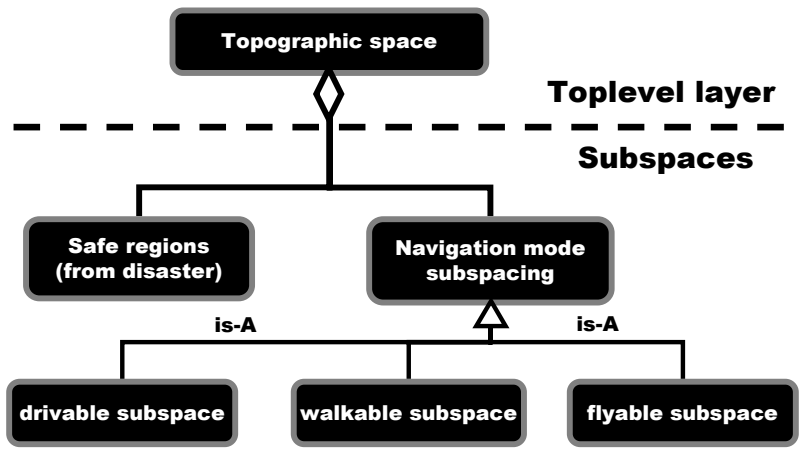

Figure 5. Some subspace layers of topographic space. The contextual subspacing of topographic space leads to space layers which are not independent from topographic space. Thus, inter-space connections are restricted to topological relations "inside" and "equals".

In fig. 6 an example for such decomposition is given. In the upper part of the figure the topographic space in terms of 5 rooms is shown. On the upper left the topographic space before and on the upper right the topographic space after the contextual selection is shown. The bases within the main topographic space are rooms and their adjacencies (e.g. connectivity) in primal and dual representation. The subspaces are represented in an own layer and permit the derivation of a trajectory for routing or guidance purposes for example in a case of a wheel chair driver (left layer in fig. 6).
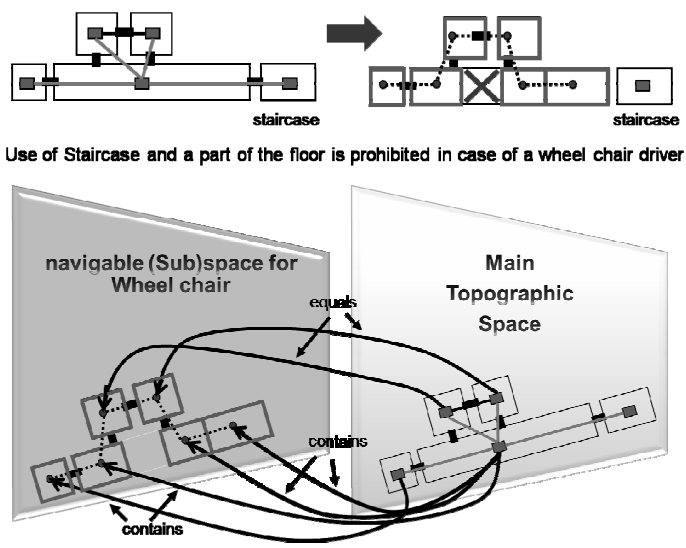

Figure 6. Example for topographic subspacing

The subspacing layer is connected to the higher level layer by the inter-space connections which express the topological relations contains or equals (see the black lines between both layers). In the case of a wheel chair driver all the possibilities to drive within the space are modeled (left layer within figure 6). Since this is a special context within navigation or routing application, this 
mode of navigation has to be modeled separately from the general topographic model. As a possible selection result from the general topographic model the upper right model can be derived. Since the topographic space is partitioned (in case of a step inside the main floor) in smaller parts only a subset of the main floor is usable for the navigation of a wheel chair driver. And finally the use of the stairs is also not possible (missing edge between last space and staircase in the upper right part of the figure).

\section{The Data Model}

The UML diagram depicted in fig. 7 shows the data model for the multilayered space model. The data model defines the classes and relations needed to describe the geometric and topological representations of each layer in primal space and their corresponding mapping in dual space. Furthermore, it contains classes representing the resulting state-transition diagram of each layer, and the npartite graph linking the states between different layers. The classes in fig. 7 are arranged according to the subdivision of each layer into four distinct regions as discussed in section 2 (see fig. 1). For each layer, its geometry and topology representations in primal and dual space are modeled in accordance with ISO 19107. Thus, the geometry representation in primal Euclidean space is represented as GM_Solids and the corresponding induced topology (natural topology, cf. Munkres [8]) as TP_Solids. Both representations are linked by "realization" associations. Following the Poincare duality, the TP Solids and $T P$ Faces in primal topology space are mapped to $T P \_$Nodes respectively $T P \_$Edges in dual space.

The resulting dual graph is equivalent to the topological part of the Node-Relation-Structure (NRS) proposed by Lee $[2,3]$ which allows for a simplified representation of the complex topological relationships between 3D spatial objects. The Euclidean space embedding of the NRS is realized as GM_Points and GM_Curves.

The separate layers of the multilayered space model are represented by the class Layer which may denote its relation to other Layers, e.g. correlation, dependency, or aggregation (for subspaces), using the association class TypeOfRelation. A Layer aggregates States and Transitions which are directly associated with the corresponding topology classes of the NRS. By this means, the statetransition diagram for each layer is realized. Layers can be connected through the InterSpaceConnection class which represents a TP_Edge in dual topology space connecting two states from separate layers. The inter-space connections (InterSpaceConnections) together with the intra-space connections (Transitions) finally establish the n-partite-Graph (Class).

The classes Space and SpaceBoundary represent real world objects in accordance with the notion of geographic features defined by ISO 19109. A Space is a semantic class corresponding to one cell in Euclidean primal space of one layer. Accordingly, SpaceBoundary is used to semantically describe the boundary faces of each cell. Both classes are seen as interfaces which connect the multilayered space model to existing semantic 3D models describing e.g. the topographic interior built environment. For example, the class Space can be related to a Room in CityGML [11] or an IfcSpace in IFC [12].

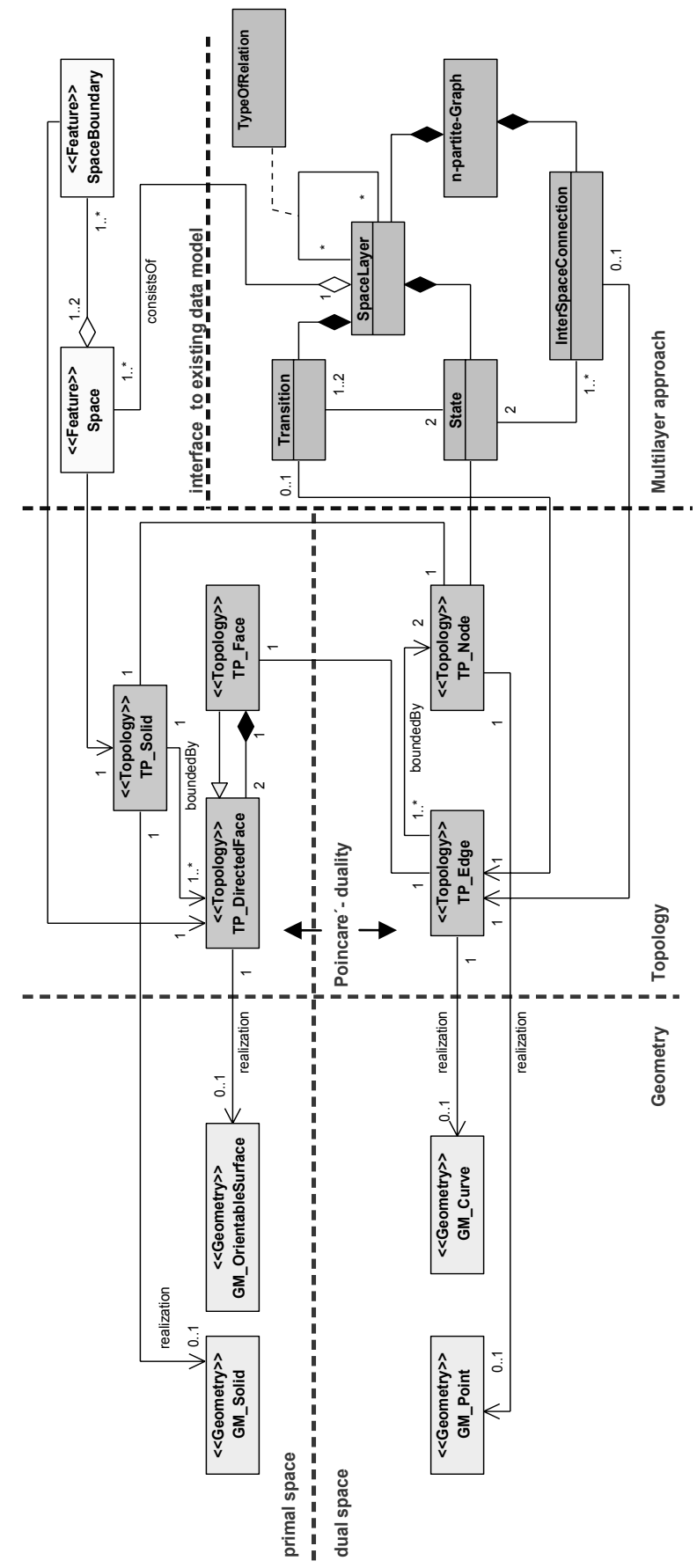

Figure 7. UML-Diagram for the modular framework 
Accordingly, SpaceBoundary may be associated with a BoundarySurface in CityGML to denote the InteriorWallSurfaces, CeilingSurfaces and FloorSurfaces of a Room, or to an IfcRelSpaceBoundary element in IFC. Furthermore, the concept of Spaces and SpaceBoundaries can be used to represent navigable and non-navigable indoor spaces. The data model does not have to be fully populated in order to derive a data structure suitable for indoor navigation systems. For example, if a 3D model of the interior built environment is not available, the geometric embedding of the topographic space layer can be omitted. However, the NRS in dual space can still be established, e.g. by measurements and observations.

As discussed in section 4, different selections of subsets of layers result in different n-partite graph structures which are suited for different aspects of indoor navigation. For example, the graph derived from a selection of the topographic space layer and specific subspaces can be used for route planning purposes. Localization and tracking can be addressed with a graph resulting from a selection of the topographic space layer and all sensor space layers in a specific navigation context.

\section{Conclusion and Outlook}

In this paper we have presented a modular concept for the modelling of indoor spaces to be used for route planning and localization/tracking within indoor environments. The model extends the work of Lee et al. [2,3] on the Node Relation Structure (NRS) by the distinction of multiple space layers. The paper also extends our previous work on the multilayered space model [1] by providing a concept for context handling, subspacing, modes of navigation, and proposal of a data model based on ISO 19107 and 19109 standards. This data model will be mapped to a GML application schema in order to define an exchange format that will be called "IndoorML".

Context is reflected by modelling the different aspects of navigation as distinct subdivisions of real space in separate layers. By keeping these aspects in separate space layers, changes in one layer (like the modification of a sensor configuration or layout) do not affect the modelling of the other aspects (like 3D indoor topography). Depending on the actual use case the corresponding space layers are selected and a context dependent npartite graph is derived from the precomputable n-partite graph of all space layers. This subgraph then will be used within the concrete scenario and by mobile devices for route planning and localization/tracking.

In the future we will examine further properties of the n-partite graph and will evaluate its usability for outdoor navigation and linkage to road network models like GDF and OpenStreetMap. Also we are currently analyzing to which extent other previously published data models for indoor navigation can be derived by our framework in the sense of being more specific models. Further examinations will show how graph structures, previously proposed by other authors $[7,9,10]$ in the field of indoor navigation can be deduced from these n-partite graph structures.

Further topics are the usage of n-partite graph for sensor placement and the acquisition of the n-partite graph without having the 3D geometries by topological modelling and measurement of properties like signal coverage e.g. for a sensor space.

\section{References}

[1] Becker, T., Nagel, C., Kolbe, T. H., A Multilayered SpaceEvent Model for Navigation in Indoor Spaces, In: Lecture Notes in Geoinformation and Cartography - 3D Geo-Information Sciences, pp. 61-77 (2009), Springer Berlin, Germany

[2] Lee, J., 3D GIS for Geo-coding Human Activity in Microscale Urban Environments. In: Egenhofer, Freksa, and Miller (Eds.): GIScience 2004 , Springer

[3] Lee, J., Zlatanova, S., 2008. A 3D data model and topological analyses for emergency response in urban areas. Geospatial Information Technology for Emergency Response - Zlatanova \& Li (eds), Taylor \& Francis

[4] Boguslawski, P., Gold, C. M., 2008, Construction Operators for Modelling 3D Objects and Dual Navigation In: Lecture Notes in Geoinformation and Cartography - 3D GeoInformation Sciences, pp. 47-59 (2009), Springer Berlin

[5] Gold, C. M., Ledoux, H., 2007. Simultaneous Storage of Primal and Dual Three-Dimensional Subdivisions. In: Computers, Environment and Urban Systems - Topology and Spatial Databases, Vol. 31, Issue 4, 2007. Elsevier

[6] Egenhofer, M., Herring, J., 1990, Categorizing Binary Topological Relations between Regions, Lines and Points in Geographical Databases, NCGIA Technical Report

[7] Lorenz, B., Ohlbach, H. J., Stoffel, E.-P., Rosner, M., 2007. Towards a Semantic Spatial Model for Pedestrian Indoor Navigation. In: Advances in Conceptual Modeling - Foundations and Applications, LNCS-Volume 4802/2007, Springer, Berlin, Germany

[8] Munkres, J. R., 1984. Elements of Algebraic Topology. Addison-Wesley, Menlo Park, CA

[9] Kolodziej, K. W., Hjelm, J., 2006, Local Positioning Systems - LBS Applications and Services, Taylor \& Francis

[10] Liao, L., Fox, D., Hightower, J. Kautz, H., Schulz, D., 2003. Voronoi Tracking: Location Estimation Using Sparse and Noisy Sensor Data. In: Proc. of the International Conference on Intelligent Robots and Systems, IROS 2003, IEEE/RSJ

[11] Gröger, G., Kolbe, T. H., Czerwinski, A., Nagel, C., 2008: OpenGIS ${ }^{\circledR}$ City Geography Markup Language (CityGML) Encoding Standard. OGC Doc. No. 08-007r1

[12] Adachi, Y., Forester, J., Hyvarinen, J., Karstila, K., Liebich, T., Wix, J. 2003: Industry Foundation Classes IFC2x Edition 3, International Alliance for Interoperability, http://www.iai-international.org. 